\title{
Optimizing BAO measurements with non-linear transformations of the Lyman- $\alpha$ forest
}

\author{
Xinkang Wang, ${ }^{1,2}$ Andreu Font-Ribera ${ }^{2}$ and Uroš Seljak ${ }^{1,2}$ \\ ${ }^{1}$ Department of Physics, University of California, Berkeley, United States \\ ${ }^{2}$ Lawrence Berkeley National Laboratory, Berkeley, United States \\ E-mail: xinkang.wang@berkeley.edu, afont@lbl.gov, useljak@berkeley.edu
}

\begin{abstract}
We explore the effect of applying a non-linear transformation to the Lyman- $\alpha$ forest transmitted flux $F=e^{-\tau}$ and the ability of analytic models to predict the resulting clustering amplitude. Both the large-scale bias of the transformed field (signal) and the amplitude of small scale fluctuations (noise) can be arbitrarily modified, but we were unable to find a transformation that increases significantly the signal-to-noise ratio on large scales using Taylor expansion up to the third order. In particular, however, we achieve a $33 \%$ improvement in signal to noise for Gaussianized field in transverse direction. On the other hand, we explore an analytic model for the large-scale biasing of the Ly $\alpha$ forest, and present an extension of this model to describe the biasing of the transformed fields. Using hydrodynamic simulations we show that the model works best to describe the biasing with respect to velocity gradients, but is less successful in predicting the biasing with respect to large-scale density fluctuations, especially for very nonlinear transformations.
\end{abstract}

Keywords: Large scale structure, Lyman- $\alpha$ forest, Baryon acoustic oscillations 


\section{Contents}

1 Introduction $\quad 1$

2 Signal-noise ratio $(\mathrm{S} / \mathrm{N})$ in a BAO measurement 2

2.1 Signal: large scale biasing 3

2.2 Noise: 1D power spectrum 3

2.3 Numerical simulations 4

$\begin{array}{lll}3 & \mathrm{~S} / \mathrm{N} \text { of non-linear transformations } & 6\end{array}$

3.1 Gaussianized field 6

$\begin{array}{lll}3.2 & \text { Generic analytic transformations } & 7\end{array}$

3.2.1 Results at second order 8

$\begin{array}{ll}3.2 .2 & \text { Results at third order }\end{array}$

4 Analytic model for large-scale bias $\quad 10$

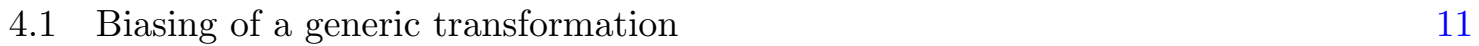

4.2 Bias computation with Fluctuating Gunn-Peterson Approximation 11

4.3 Testing the model with simulations 12

5 Conclusions $\quad 14$

$\begin{array}{lr}\text { A Bias fitting procedures } & 15\end{array}$

B Large-scale bias for $\mathrm{z}=2.4$ and $2.75 \quad 16$

\section{Introduction}

One of the main drivers of current cosmological observations is the study of the accelerated expansion of the Universe [1]. One particular probe of the acceleration has recently proven very successful: the measurement of the Baryon Acoustic Oscillation (BAO) scale in the clustering of galaxies, which can be used as a cosmic ruler to study the geometry of the Universe at different redshifts [2]. Even though the first measurements of the BAO scale were presented in the context of galaxy clustering at low redshift [3-6], in theory any tracer of the large-scale distribution of matter could be used to measure BAO. At high redshift $(z>2)$, for instance, the $\mathrm{BAO}$ scale was recently measured in the clustering of the absorption features in distant quasar spectra [7-10], a technique known as the Lyman- $\alpha$ forest, or Ly $\alpha$ forest.

As light emitted from distant quasars travels through an expanding Universe, it is gradually redshifted towards longer wavelengths. Photons that have escaped from the quasars with energy above the Ly $\alpha$ transition energy might be absorbed by neutral hydrogen at positions where the redshifted wavelength of the photons equals that of the Ly $\alpha$ transition energy. Hence, photons emitted with different energies from a quasar will have to travel through different distances to be absorbed (when they reach the Ly $\alpha$ transition wavelength), and as a consequence the fraction of transmitted flux for a given photon wavelength is a function of the neutral hydrogen density, which in turn is a function of line-of-sight position from the quasar $[11,12]$. Although the distribution of neutral hydrogen on very small scales 
(e.g., a few kpc) is determined by the thermal properties of intergalactic medium, on cosmological scales it can be described as a tracer of the underlying density field, which was confirmed in the first studies of the clustering of the absorption features in high resolution spectra from a handful of bright quasars [13-15]. Since then, Ly $\alpha$ forest has become an increasingly important tool to study the large-scale structure of the Universe, and up to now it is considered one of the most promising methods to study the clustering on both the smallest scales (via the correlated absorption along a single line of sight) and on the largest scales (via the correlations in neighboring lines of sight).

An interesting question that we will address in this paper is whether the information extracted from a Ly $\alpha$ forest survey can be increased by a nonlinear transformation of the observed field. Similar approaches have been suggested in the context of weak lensing and galaxy clustering [16-19]. In [20], it was suggested that one could increase the signal-to-noise ratio of a measurement by applying proper non-linear transformation to the Ly $\alpha$ transmitted flux fraction (i.e., $F \rightarrow g(F)$ ). In this paper, in particular, we study whether such transformations could improve the BAO measurement from the Ly $\alpha$ forest. We will also compare the analytic predictions of $\operatorname{Ly} \alpha$ forest bias in [20] (for both the flux field and the transformed fields) to simulations.

Specifically, the performance of a Ly $\alpha$ forest BAO survey can be quantified by the error bars in the measurements of the BAO scale along and across the line of sight, respectively, as well as their correlation coefficient. In order to optimize this measurement, we would like to have realistic simulations of a hypothetical survey and directly study the effect of different transformations on the error bars of the measured BAO scales. Unfortunately, this is beyond the reach of current computational facilities. Hydrodynamic simulations are thus required in order to accurately reproduce the statistics of the Ly $\alpha$ forest. However, these simulations are not able to simulate boxes large enough to cover a full BAO survey. On the other hand, in order to test the analysis pipeline and study the effect of potential systematics, current BAO analyses using the Ly $\alpha$ forest rely on simplified mocks [21, 22]. Despite the fact that these mocks have the correct 1- and 2-point correlation functions, their higher order statistics are generally not correct, and thus would not be useful for our study.

As a forecast, in section 2 we will show that under several assumptions the effect of a non-linear transformation $F \rightarrow g(F)$ boils down to a single quantity: the ratio of largescale biasing of the transformed field (squared) over the one-dimensional power spectrum of the field in large-scale limit, both of which can be measured with current hydrodynamic simulations, allowing us to study the effect of non-linear transformations on the Ly $\alpha$ forest in the context BAO measurements. We present the results of this study in section 3. In addition, in order to better understand the effect of the transformations, in section 4 we extend the bias model of [20] to describe the bias of non-linear analytic transformations. We conclude our results in section 5 .

\section{Signal-noise ratio $(\mathrm{S} / \mathrm{N})$ in a $\mathrm{BAO}$ measurement}

The main goal of a BAO survey is to provide a measurement of the BAO scale at a given redshfit, as precise as possible. In order to predict the performance of a BAO survey, as a result, we would like to generate simulations of the survey, measure its power spectrum, fit the $\mathrm{BAO}$ scale on this measurement and look at its precision. We would like the simulation to be as realistic as possible, and we would like the analysis to be similar to that used with the real data. 
In order to simulate accurately the $\operatorname{Ly} \alpha$, however, we need to use expensive hydrodynamic simulations with a really high spatial resolution [23]. This sets the maximum volume that one can simulate to roughly $100^{3} h^{-3} \mathrm{Mpc}^{3}$, clearly insufficient to measure the BAO scale. Fortunately, there are several approximations that we can use to make the problem more treatable. Specifically, if the detection of the BAO scale is statistically very significant, the variance of the BAO measurement will scale proportionally to the covariance matrix of our power spectrum measurement, relative to the signal level (the power spectrum itself). Moreover, assuming that linear theory holds on the relevant scales, the different bins in the power spectrum can be treated as independent, resulting in a diagonal covariance matrix.

We can therefore quantify the precision of a given survey with a signal-to-noise function $S / N(\mathbf{k})$, defined as the ratio of the power spectrum (signal) and its uncertainty (noise). In this section, we will present further approximations that will allow us to compress the information of this function into a single number.

\subsection{Signal: large scale biasing}

The BAO information in a Ly $\alpha$ forest survey is contained in Fourier modes with rather small wave numbers $k<0.3 \mathrm{hMpc}^{-1}$, where we expect the power spectrum $P_{F}(\mathbf{k})$ to follow a simple linear bias model:

$$
P_{F}(\mathbf{k})=b_{F}^{2}(\mu) P_{m}(k),
$$

where $P_{m}(k)$ is the matter power spectrum, $\mu=\hat{k} \cdot \hat{z}$ with $\hat{z}$ being the line-of-sight direction, and $b_{F}(\mu)$ describes the large-scale biasing of the Ly $\alpha$ forest including redshift space distortions. The angular dependence of the biasing follows the usual Kaiser formula [24]:

$$
b_{F}(\mu)=b_{F}^{\delta}+\left(f \mu^{2}\right) b_{F}^{\eta},
$$

where $f$ is the logarithmic growth rate. In particular, this growth rate is close to one in a $\Lambda \mathrm{CDM}$ universe at the redshifts of interest $(z>2)$, where the universe is close to Einstein-de Sitter, and will taken as unity from now on except stated otherwise. $b_{F}^{\delta}$ and $b_{F}^{\eta}$ are related to the response of the transmitted flux fraction $F$ to small variations of the density and of the line-of-sight velocity gradient, respectively. In section 4 we will present an analytic model to predict their values, but for now we can think of these as free parameters.

An analytic non-linear transformation of flux $F$ is an analytic function $g(F)$. Assuming linear theory holds in the relevant scales, the power spectrum of this transformed field, $P_{g}(\mathbf{k})$, can be described using the same equations 2.1 and 2.2 with $\left(b_{F}^{\delta}, b_{F}^{\eta}\right)$ replaced by a different set of bias parameters: $\left(b_{g}^{\delta}, b_{g}^{\eta}\right)$. Therefore, the impact of the transformation on the BAO signal can be described by the ratio of the bias parameters squared: $b_{g}^{2}(\mu) / b_{F}^{2}(\mu)$.

Note that most studies of the large-scale clustering of the Ly $\alpha$ forest focus on the statistics of the fluctuations around the mean transmitted flux fraction, i.e., $\delta_{F}=F /\langle F\rangle-1$. The transformed field, however, might have an arbitrarily small mean, and for simplicity we choose to focus on the clustering of the $F$ and $g(F)$ fields themselves.

\subsection{Noise: 1D power spectrum}

The error bars in the BAO measurements are set by the uncertainty with which we are able to measure the clustering on the Fourier modes relevant for BAO. Following [25], the uncertainty on a given Fourier mode is proportional to

$$
\sigma_{P_{F}}^{2}(\mathbf{k})=2\left(P_{F}(\mathbf{k})+P_{N}^{e f f}+A P_{F}^{1 D}\left(k_{\|}\right) / n_{q}^{2 D}\right)^{2} .
$$


There are three contributions to the noise: i) the first term, $P_{F}(\mathbf{k})$, is set by the signal and is usually referred as cosmic variance; ii) the second term, $P_{N}^{e f f}$, is set by the level of instrumental noise in the spectra, and is usually referred as noise power; iii) the last term, $A P_{F}^{1 D}\left(k_{\|}\right) / n_{q}^{2 D}$, takes account of the fact that we can only sample the universe in a set of thin lines of sight, and is thus set both by the level of intrinsic fluctuations (or 1D power $\left.P^{1 D}\left(k_{\|}\right)\right)$on a scale $\sim k_{\|}=k \mu$ and by the $2 \mathrm{D}$ density of background sources $n_{q}^{2 D}{ }^{1}$. This last term is usually referred as aliasing noise and is equivalent to the shot noise in galaxy clustering studies. Now, as noted in [26], $P_{F}^{1 D}\left(k_{\|}\right)$is approximately flat on scales relevant for $\mathrm{BAO}$ analyses. Since the noise power is also flat (white noise), the last two terms in eq.2.3 can thus be described by a single term $P_{F}^{1 D}\left(k_{0}\right) / n_{\text {eff }}$, where $P_{F}^{1 D}\left(k_{0}\right)$ describes the typical level of $P_{F}^{1 D}\left(k_{\|}\right)$in the flat regime (i.e., $k_{0}$ large enough) and $n_{e f f}$ is an effective density of lines of sight that takes into account the distribution of noise power in the spectra.

In present and near-future $\mathrm{Ly} \alpha$ forest BAO surveys, the contribution from cosmic variance is rather small, and we will ignore it in this study. The other two contributions are comparable, but for simplicity in this study we will only consider the aliasing term. And since the instrumental noise is very close to Gaussian, any non-linear transformation applied to the observed flux field will make the noise properties non-Gaussian. By ignoring the noise in our analysis, we are simplifying the problem, and our results should be read as the most optimistic case in the limit of low noise. We will thus characterize the effect of the non-linear transformation on the BAO uncertainties with the ratio of the amplitudes of the one-dimensional power in small $k_{\|}$limit: $P_{g}^{1 D}\left(k_{0}\right) / P_{F}^{1 D}\left(k_{0}\right)$.

After all, for any non-linear transformation $F \rightarrow g(F)$, we expect the following proportionality holds on large scales:

$$
S / N(\mathbf{k}) \propto R_{g}(\mu)=b_{g}^{2}(\mu) / P_{g}^{1 D}\left(k_{0}\right) .
$$

The right-hand side of this equation may serve as a proxy for $\mathrm{S} / \mathrm{N}$ and is a measurable quantity in simulations. We will focus on the value of $R_{g}$ in the rest of the paper.

\section{$2.3 \quad$ Numerical simulations}

In next section we will study the effect of analytic non-linear transformations $g(F)$ on the signal-to-noise ratio $(\mathrm{S} / \mathrm{N})$ with which we can measure BAO. To do that we will use hydrodynamic simulations to measure the large-scale biasing (squared) of the transformed field, $b_{g}^{2}(\mu)$ (proxy for signal), and to measure the 1D power spectrum of the transformed in low- $k$ limit, $P_{g}^{1 D}\left(k_{0}\right)$ (proxy for noise).

The simulation we use is one of the Gadget-3 simulations adopted in the Nyx/Gadget-3 comparison project [27], with a box size of $40 h^{-1} \mathrm{Mpc}$ and a total of $2 \times 1024^{3}$ particles. The simulation used a WMAP7 cosmology $\left(\Omega_{b}=0.046, \Omega_{m}=0.0275, \Omega_{\Lambda}=0.725, h=0.702\right.$, $\sigma_{8}=0.816$, and $\left.n_{s}=0.96\right)$ and the ionizing background prescription of [28]; it also used the QUICKLYA option in Gadget to implement a simple star formation recipe. We have rescaled the optical depth in the box in order to have a mean flux of $\langle F\rangle=0.8413$ (at $z=2$ ), in agreement with estimates from SDSS [29]. In the main part of this paper we use only the simulation output at $z=2$, but in appendix B we also present results using outputs at redshift $z=2.4$ and $z=2.75$.

\footnotetext{
${ }^{1}$ The constant $A$ depends on the distribution of weights in the different spectra, but for a given survey this can be treated as a constant
} 


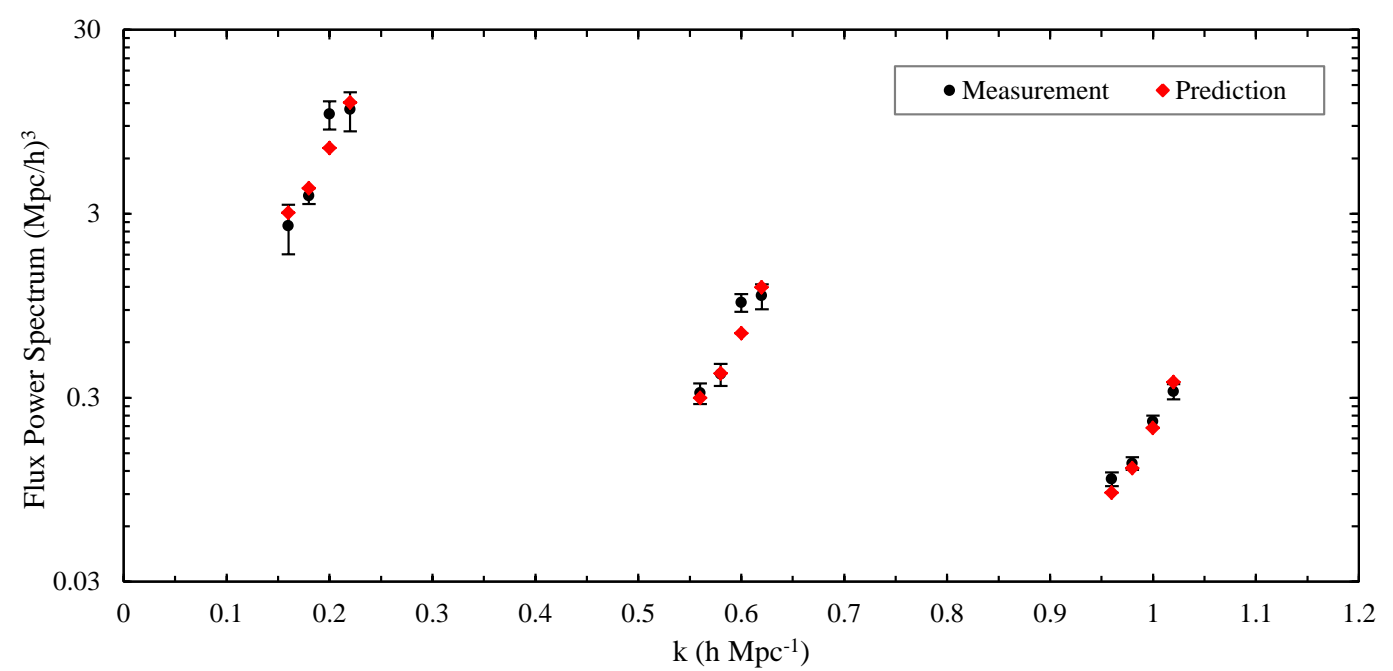

Figure 1. The plotted scales are $k=0.2,0.6,1.0 \mathrm{~h} \mathrm{Mpc}^{-1}$, each of which has four $\mu$ bins. For clarity, at each $k$ the power spectrum with $\mu=0.125$ is plotted at $(k-0.04), \mu=0.375$ is plotted at $(k-0.02)$, $\mu=0.625$ at $k$, and $\mu=0.875$ at $(k+0.02)$.

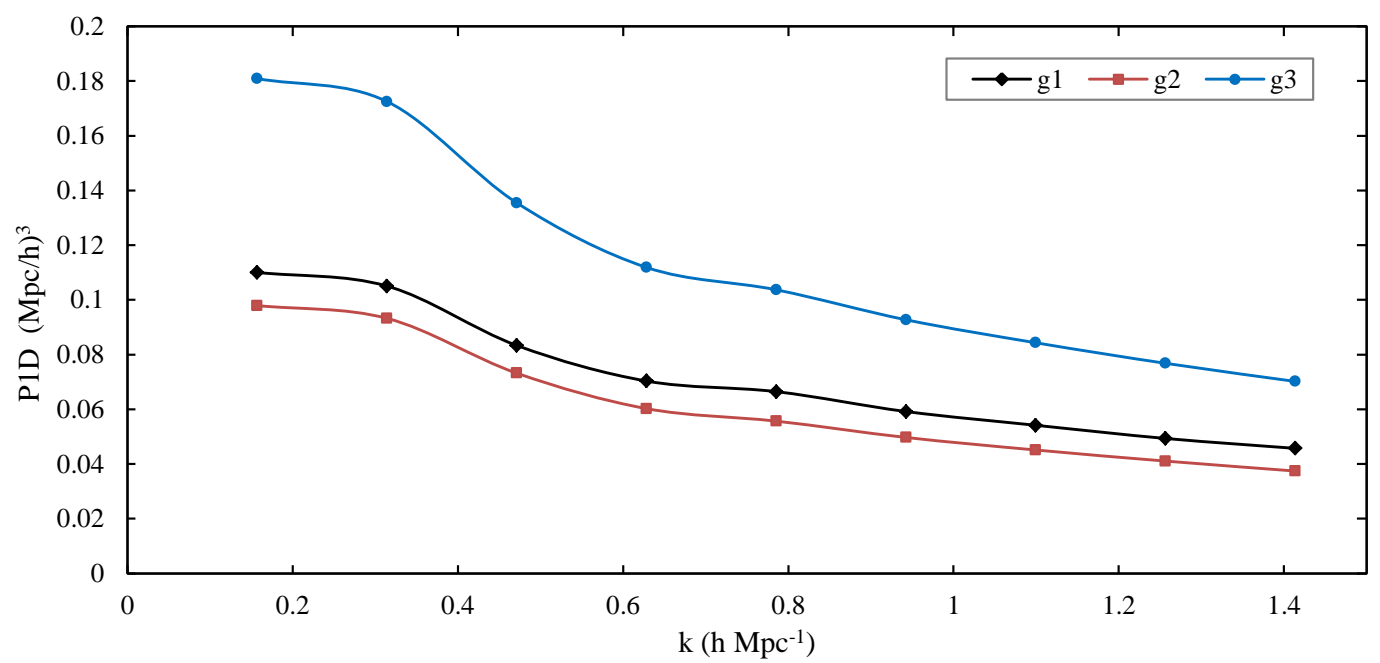

Figure 2. 1D power spectrum measured in simulations for the original field $g_{1}(F)=F$ (black line), and the two other fields discussed in the next section. We use the 1D power in the fundamental mode of the simulation $\left(k_{0}=0.157 h \mathrm{Mpc}^{-1}\right)$ as a proxy for the noise in a BAO measurement.

We now show that the proxies for signal and noise we chose in previous two subsections (i.e., eq.(2.4)) are valid, respectively. In figure 1, we show (in black) the 3D power spectrum of the transmitted flux fraction $F$, measured at three different scales $k=0.2,0.6,1.0 \mathrm{hMpc}^{-1}$, and at four different line-of-sight directions $(\mu=0.125,0.375,0.625,0.875)$. The red points show the prediction using eq.2.1 for the values of $\left(b_{F}^{\delta}=-0.0733, b_{F}^{\eta}=-0.0995\right)$ that best fit the simulations (see appendix A for a description of how we fit the bias parameters in simulations). The figure clearly shows the validity of squared bias as the proxy for signal. On the other hand, it is important to remind the reader that throughout this paper, we will not follow the common convention of measuring statistics of fields that have been normalized (i.e., divided by its mean). Therefore, if one wishes to compare the flux biases reported in this paper, one would have to divide them by the mean flux in the simulation: $\langle F\rangle=0.8413$. 
In figure 2 we show the measured 1D power spectrum of the transmitted flux fraction $g_{1}(F)=F$, and two of the transformed fields discussed in next section: $g_{2}(F)=-0.261 F+F^{2}$ and $g_{3}(F)=1.084 F-3.039 F^{2}+F^{3}$. In the figure it is clear that $P_{g}^{1 D}(k)$ is close to constant at scales relevant in BAO analyses $\left(k<0.3 h \mathrm{Mpc}^{-1}\right)$. We will use the $1 \mathrm{D}$ power at the fundamental mode, $k_{0}=0.1571 \mathrm{hMpc}^{-1}$, as a proxy for the noise in a BAO measurement.

\section{$3 \mathrm{~S} / \mathrm{N}$ of non-linear transformations}

In the previous section we have shown that under several assumptions, the performance of a BAO survey can be quantified using the ratio of the large-scale bias (squared) over the low $k$ limit of the one dimensional power spectrum. We also presented the measurement of these quantities in the original Ly $\alpha$ absorption field using hydrodynamic simulations. In this section, we will use the same simulations to measure the equivalent quantities in different fields which are obtained by performing non-linear transformations to the original field.

\subsection{Gaussianized field}

A Gaussian field can be fully described by its 2-point correlation function. The Ly $\alpha$ forest flux field, on the other hand, is highly non-Gaussian, implying that in order to obtain all of its cosmological information we would need to use higher order statistics. Therefore, it is tempting to transform the flux field to make it as Gaussian as possible [13, 30]. For instance, we can assume a monotonic relation between our flux field $F$ and a Gaussianized field $g(F)$, which is implicitly defined via

$$
\int_{-\infty}^{g(F)} d g^{\prime} p_{g}\left(g^{\prime}\right)=\int_{0}^{F} d F^{\prime} p_{F}\left(F^{\prime}\right)
$$

where $p_{g}(g)$ and $p_{F}(F)$ are the probability distribution function (PDF) of each field. After measuring the PDF of flux in simulations, $p_{F}(F)$ (shown in figure 6), we are able to determine the monotonic function $g(F)$ and transform the flux into a Gaussianized field, with zero mean and unit variance. In figure 3 we show the transformation measured at different redshifts. We apply such transformation to the flux field in simulation and measure the $\mathrm{S} / \mathrm{N}$ (i.e., $R_{g}(\mu)$ in eq.(2.4)) of the resulting Gaussianized field.

We measure the one-dimensional power of the Gaussianized field, $P_{g}^{1 D}\left(k_{0}\right)$, to be $19.20 \pm$ 0.05 times higher than that of the original field $F$. On the other hand, the bias parameters of the Gaussianized field are also larger, by a factor of $5.06 \pm 0.32$ for $b_{g}^{\delta}$ and a factor of $2.70 \pm 0.41$ for $b_{g}^{\eta}$. Interestingly, this result implies a very anisotropic gain in signal-to-noise ratio: while the $\mathrm{S} / \mathrm{N}$ transverse to the line of sight $(\mu=0)$ is measured a factor of $1.33 \pm 0.17$ larger, the $\mathrm{S} / \mathrm{N}$ along the line of sight $(\mu=1)$ is measured a factor of $0.71 \pm 0.08$ smaller. In addition, in large scale structure analyses it is common to define a redshift space distortion parameter $\beta=f b_{\eta} / b_{\delta}$. In our simulations we measure it to be roughly $\beta_{F}=1.30$ for the original flux field $F$. The Gaussianized field $g(F)$ described above, however, has weaker redshift space distortions with only $\beta_{g}=0.69$, where we have used growth rate $f=0.96$.

Finally, since standard Ly $\alpha$ forest BAO measurements preferentially measure the lineof-sight scale $[7,9,10]$, with very large uncertainties in the transverse direction, the fact that the measurement in the transformed Gaussianized field favors the transverse direction might be of special interest. 


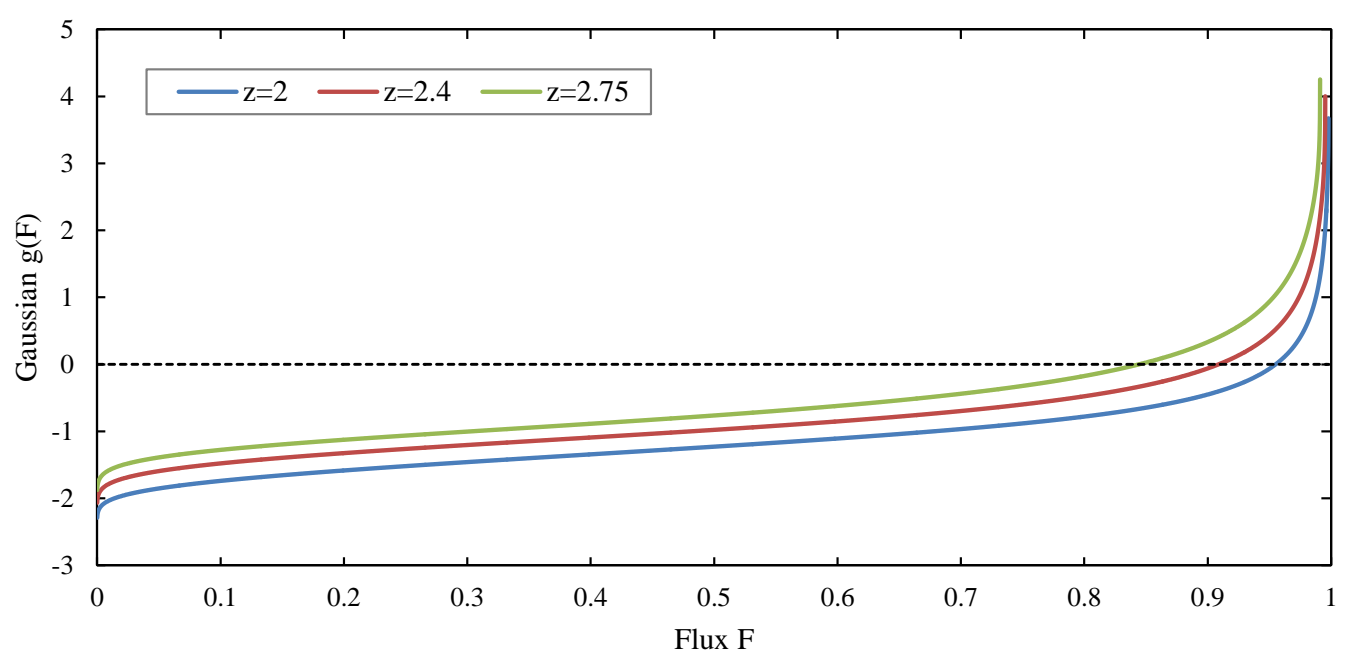

Figure 3. Monotonical relation $g(F)$ to convert the simulated flux field into a Gaussianized field with zero mean and unit variance. The different lines correspond to $z=2$ (blue), $z=2.4$ (red) and $z=2.75$ (green). The dashed line (black) is the "zero" reference line.

\subsection{Generic analytic transformations}

The ultimate goal of this paper is to find an analytic non-linear transformation that maximally enhances $\mathrm{S} / \mathrm{N}$ with respect to that of the orignal flux field. To achieve this goal, we need to consider the entire set of generic analytic transformations $g(F)$ which can be expressed as an infinite Maclaurin series:

$$
g(F)=\sum_{m=1}^{\infty} a_{m} F^{m}, \quad a_{m}=\frac{g^{(m)}(0)}{m !} .
$$

In practice, however, we will study only the transformations involving terms up to $F^{n}\left(a_{n} \neq\right.$ 0 ), and refer to these as transformations of order $n$. Notice that we have ingored the zeroth order in the series, of which the specific value has no effect on the following study.

We can think of $F^{m}$ as another tracer of the density field, with bias parameters $b_{F^{m}}(\mu)=$ $b_{F m}^{\delta}+\left(f \mu^{2}\right) b_{F m}^{\eta}$. In linear regime, the large-scale cross-correlation of two tracers is just proportional to the product of their bias parameters, and therefore the cross-power spectrum can be expressed as the geometric mean of their power spectra. This allows us to define the biasing of the transformed field $g(F)$ :

$$
b_{g}(\mu)=\sum_{m=1}^{n} a_{m} b_{F^{m}}(\mu)=\sum_{m=1}^{n} a_{m}\left(b_{F^{m}}^{\delta}+\left(f \mu^{2}\right) b_{F^{m}}^{\eta}\right)=b_{g}^{\delta}+\left(f \mu^{2}\right) b_{g}^{\eta}
$$

On the other hand, for the noise, the one-dimensional power spectrum $P^{1 D}\left(k_{\|}\right)$is related to the three-dimensional power spectrum $P\left(k_{\|}, \mathbf{k}_{\perp}\right)$ by an integral over $\mathbf{k}_{\perp}$. Even in the limit of small $k_{\|}$, the $1 \mathrm{D}$ power is affected by high $\mathbf{k}_{\perp}$ modes that are not well described by linear theory. This implies that the $1 \mathrm{D}$ power is not directly proportional to the bias parameters, and that the 1D cross-power of two fields $P_{\left(F^{m}, F^{i}\right)}^{1 D}$ can not described by the geometric mean of their 1D powers. Therefore, if we are interested in studying generic transformations up to a certain order $n$, we have to measure not only all the $1 \mathrm{D}$ power spectra $P_{F^{j}}^{1 D}\left(k_{0}\right)$ where 
$j \leq n$, but also all the $1 \mathrm{D}$ cross-power spectra $P_{\left(F^{j}, F^{k}\right)}^{1 D}\left(k_{0}\right)$ :

$$
P_{g}^{1 D}\left(k_{0}\right)=\sum_{m=2}^{n} \sum_{i=1}^{m-1} a_{i} a_{m-i} P_{\left(F^{i}, F^{m-i}\right)}^{1 D}\left(k_{0}\right),
$$

where $P_{\left(F^{i}, F^{m-i}\right)}^{1 D}=P_{F^{i}}^{1 D}$ if $i=m-i$. Notice that $k_{0}$ is the fundamental mode.

To compute $\mathrm{S} / \mathrm{N}$ for $g(F)$ of order $n$, we can compute in simulations the relevant biases $b_{F^{m}}($ for $m \leq n)$ and 1D auto- and cross-power spectra $P_{\left(F^{m}, F^{i}\right)}^{1 D}\left(k_{0}\right)$. Moreover, multiplying the field by a constant would have no effect on the signal-to-noise ratio, since both quantities would be modified by the same multiplicative factor. Therefore, we are only interested in $g(F)$ of the monic form: $g(F)=q_{1} F+q_{2} F^{2}+\cdots+q_{n-1} F^{n-1}+F^{n}$, where $q_{i}=a_{i} / a_{n}$.

Even though the signal is anisotropic, we can compute an angularly average signal (the power spectrum monopole), and use a single averaged bias parameter to quantify the signal:

$$
\bar{b}_{g}^{2}=\int_{0}^{1} d \mu b_{g}^{2}(\mu)=\left(b_{g}^{\delta}\right)^{2}+\frac{2}{3} b_{g}^{\delta} b_{g}^{\eta}+\frac{1}{5}\left(b_{g}^{\eta}\right)^{2} .
$$

Finally, we have an averaged version of $\mathrm{S} / \mathrm{N}$ for any $n^{\text {th }}$ order generic transformation:

$$
\bar{R}_{g}=\frac{\bar{b}_{g}^{2}}{P_{g}^{1 D}\left(k_{0}\right)}=f\left(q_{1}, q_{2}, \ldots, q_{n-1}\right) .
$$

The functional form $f$ is to indicate there are only $n-1$ variables we need to consider for $n^{\text {th }}$ order $g(F)$. Most importantly, at any given order, by maximizing $\bar{R}_{g}$ we can find the transformation that should result in a larger gain in signal-to-noise ratio. We will compare the results to the value of $\bar{R}_{g}$ for the original flux field: $\bar{R}_{F}=0.1109$.

\subsubsection{Results at second order}

We consider an arbitrary quadratic field $g(F)=q_{1} F+F^{2}$, which only contains a single parameter $q_{1}$. Using the measured bias values $\left(b_{F}^{\delta}, b_{F}^{\eta}, b_{F^{2}}^{\delta}, b_{F^{2}}^{\eta}\right), 1 \mathrm{D}$ power $\left(P_{F}^{1 D}\left(k_{0}\right), P_{F^{2}}^{1 D}\left(k_{0}\right)\right)$ and cross-power $\left(P_{\left(F, F^{2}\right)}^{1 D}\left(k_{0}\right)\right)$, we can estimate the angularly averaged signal-to-noise ratio function, $f\left(q_{1}\right)$, and numerically/analytically find the global maximum of this function.

The signal-to-noise ratio function $f\left(q_{1}\right)$ for a generic quadratic transformation is shown in figure 4 (in black), in which two different angular configurations are also shown for comparison: $R_{g}(\mu=1)$ along the line of sight (in red), and $R_{g}(\mu=0)$ across the transverse direction (in blue). As expected, for large absolute values of $q_{1}$ we recover the signal-to-noise ratios of the original field $F$ (dashed lines). We find that the angularly averaged function has a global maximum at $q_{1}^{(1)}=-0.261$, where $\bar{R}_{g}=f\left(q_{1}^{(1)}\right)=0.1147$, and the global minimum is at $q_{1}^{(2)}=-1.22$, where the signal drops close to zero. The zero signal is due to the fact that the signal scales as $\left(q_{1} b_{F}^{\delta}+b_{F^{2}}^{\delta}\right)$, and with $b_{F}^{\delta}, b_{F^{2}}^{2}$ fixed there is always a $q_{1}$ at which the signal vanishes. Similar argument applies to the higher order case shown in figure 5 .

In the previous section we saw that when the flux field is Gaussianized, the $\mathrm{S} / \mathrm{N}$ gain with respect to that of the original flux field varies anisotropically. Interestingly, this is not the case for a generic quadratic transformation, since the three gains in figure 4 are remarkably similar. One may wonder why. The angular dependence of the signal is set by the redshift space distortion parameter of the field, $\beta_{g}=f b_{g}^{\eta} / b_{g}^{\delta}$. In our simulations we find that $\beta_{F^{2}} \sim \beta_{F}$, and therefore any linear combination of these fields will have also a similar $\beta_{g}$, which explains the weak angular dependence of the $\mathrm{S} / \mathrm{N}$ gain. 


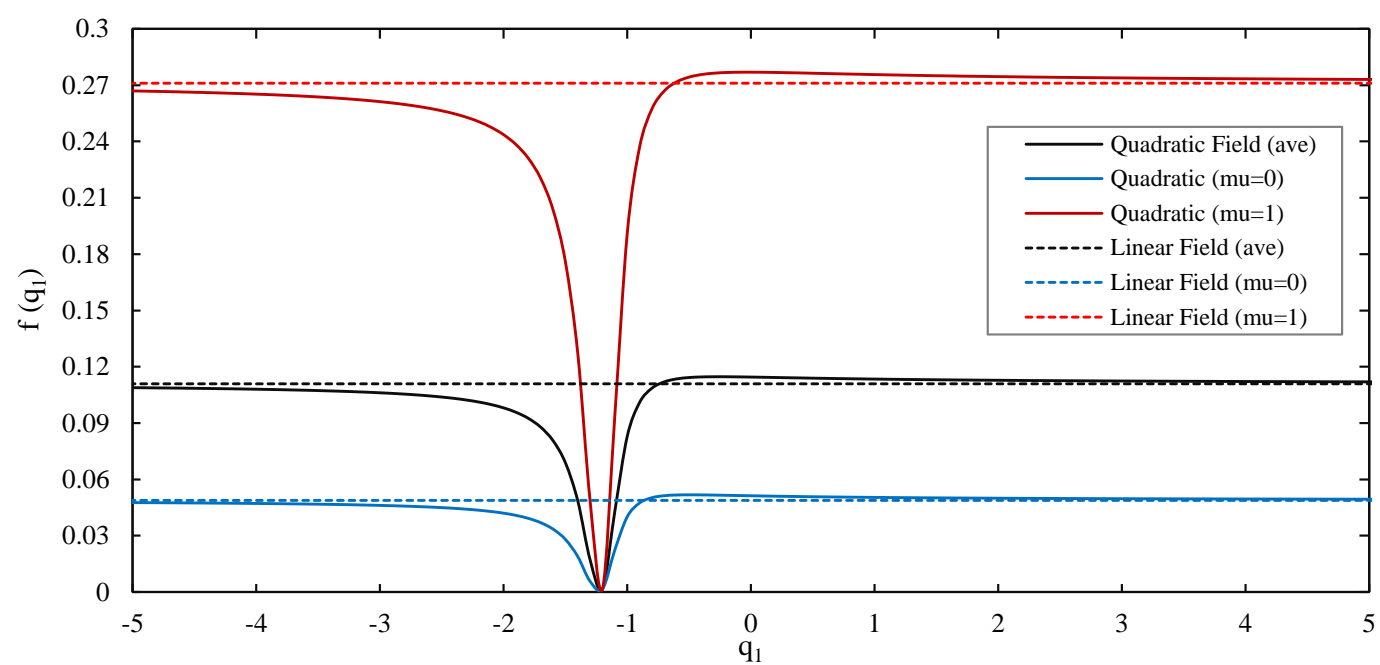

Figure 4. Signal-to-noise ratio for generic quadratic transformations $g(F)=q_{1} F+F^{2}$, as a function of $q_{1}$. The line-of-sight direction $(\mu=1)$ is shown in red, the transverse direction $(\mu=0)$ is shown in blue and the angular average (equation 3.6) is shown in black. For large absolute values of $q_{1}$, we recover the signal-to-noise ratios of the original field $F$ (dashed lines).

Finally, we note that even at the global maximum $q_{1}^{(1)}$, the predicted signal-to-noise ratio is only $3.40 \%$ larger than that of the original field. To test this prediction, we have applied the transformation $g_{2}(F)=-0.261 F+F^{2}$ to the flux field in the simulations and have measured the statistics of the transformed field. The measured signal-to-noise ratio is $(3.56 \pm 8.74) \%$ larger than the reference value, consistent with our prediction. The uncertainty in the quoted ratio is set by the uncertainty in our measurement of the bias parameters.

\subsubsection{Results at third order}

We consider now generic cubic transformations $g(F)=q_{1} F+q_{2} F^{2}+F^{3}$. After measuring the bias parameters for $F^{3}$, its 1D power spectrum and its cross-power spectra with $F$ and $F^{2}$, we can extend the previous study on quadratic fields to include transformations of order $n=3$.

In figure 5 we show the angularly averaged signal-to-noise ratio for generic cubic transformations. The global maximum corresponds to the transformation $g_{3}(F)=1.084 F-$ $3.039 F^{2}+F^{3}$, where $\bar{R}_{g}$ is only $3.47 \%$ larger than that of the original $F$ field. We have also tested this in simulations, by applying the transformation $g_{3}(F)$ to the flux field and measuring its statistics. The gain measured in simulations is $(4.09 \pm 8.79) \%$.

We have explored higher order transformations, but the results become numerically very unstable, and we are not able to reproduce the predicted $\bar{R}_{g}$ in simulations. For instance, the noise term for $n=4$ involves a total of ten 1D auto- and cross-power spectra, each of which has an associated numerical uncertainty. We have also repeated the analyses at different redshift outputs $(z=2.4$ and $z=2.75)$, finding results qualitatively similar.

Finally, in table 1 we list several relevant quantities measured in the simulations for some of the fields mentioned in this section, including the quadratic and the cubic transformations with higher angularly averaged signal-to-noise ratio, as well as the Gaussianized field. Note that we cannot exclude that there may be other transformations that give a better improvement than the ones we explored here: the fact that we do not approach the gains 


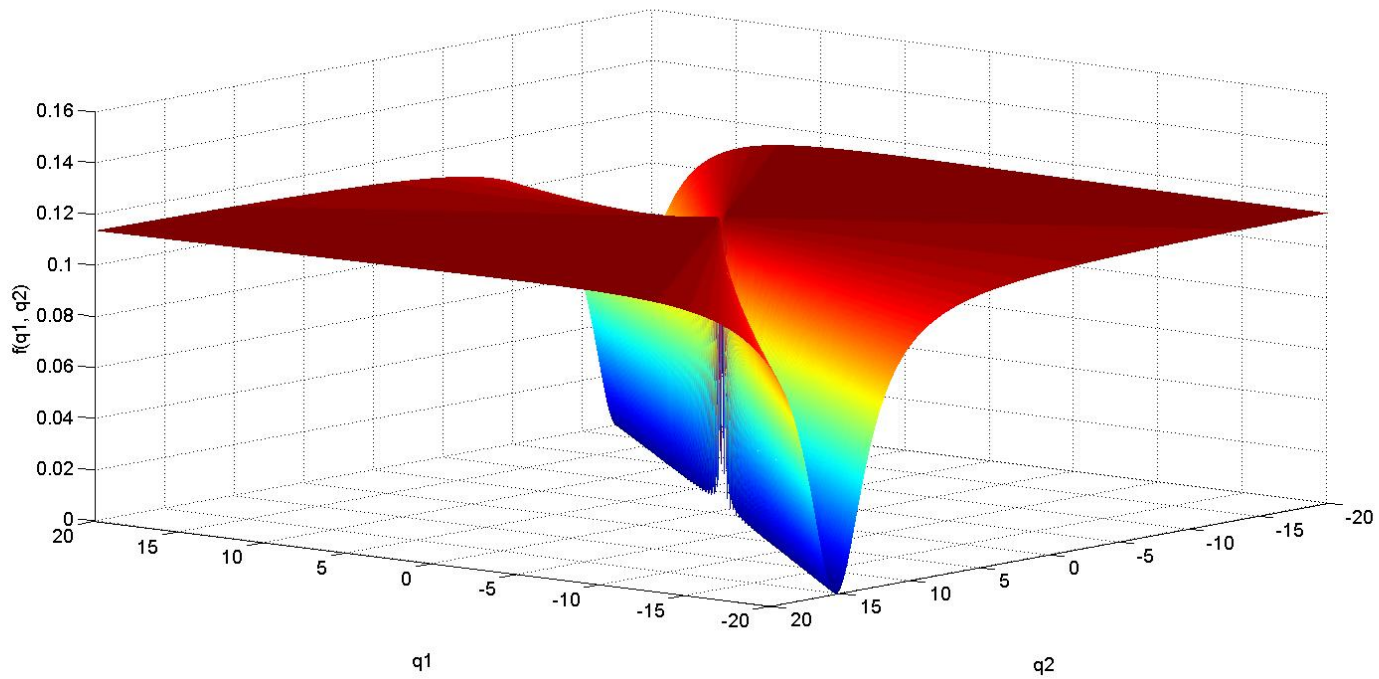

Figure 5. Angularly averaged signal-to-noise ratio for generic cubic transformations, as a function of $q_{1}$ and $q_{2}$.

\begin{tabular}{|c||c|c|c|c|c||c|c|c|}
\hline$g$-field & $\langle g\rangle$ & $b_{g}^{\delta}$ & $b_{g}^{\eta}$ & $\beta_{g}$ & $P_{g}^{1 D}\left(k_{0}\right)$ & $\bar{R}_{g}$ & $R_{g}(\mu=0)$ & $R_{g}(\mu=1)$ \\
\hline$F$ & 0.8413 & -0.0733 & -0.0994 & 1.302 & 0.1101 & 0.1109 & 0.0487 & 0.2710 \\
\hline$F^{2}$ & 0.7703 & -0.0902 & -0.1194 & 1.271 & 0.1587 & 0.1145 & 0.0512 & 0.2769 \\
\hline$F^{3}$ & 0.7197 & -0.0994 & -0.1248 & 1.205 & 0.1861 & 0.1143 & 0.0531 & 0.2701 \\
\hline$g_{2}(F)$ & 0.5509 & -0.0712 & -0.0935 & 1.261 & 0.0980 & 0.1148 & 0.0517 & 0.2768 \\
\hline$g_{3}(F)$ & -0.7094 & 0.0957 & 0.1302 & 1.306 & 0.1809 & 0.1154 & 0.0507 & 0.2824 \\
\hline Gaussianized & 0.0080 & -0.3706 & -0.2679 & 0.694 & 2.1132 & 0.1031 & 0.0650 & 0.1929 \\
\hline \hline
\end{tabular}

Table 1. Summary of values used in this section (measured directly from simulation). For each field $g$, we present its mean $\langle g\rangle$, its density bias $b_{g}^{\delta}$, velocity bias $b_{g}^{\eta}$, redshift space distortion parameter $\beta_{g}, 1 \mathrm{D}$ power $P_{g}^{1 D}\left(k_{0}\right)$ in low- $k$ limit, angularly averaged signal-to-noise ratio $\bar{R}_{g}$, transverse ratio $R_{g}(\mu=0)$ and line-of-sight ratio $R_{g}(\mu=1)$. The simulation we use is not able to determine the correct sign of bias by itself, and therefore for consistency we set bias negative for $g(F)$ with positive mean $\langle g\rangle$ and vice versa. In particular, to compute $\beta$ we have used $f=0.96$ for precision.

achieved by Gaussianized field for $\mu=0$ by the third order expansion suggests that it may be possible to find other transformations that offer large gains, but the Taylor expansion approach used here does not seem to find them at the order we are working.

\section{Analytic model for large-scale bias}

Paper [20] presents an analytic model to describe the biasing of the Ly $\alpha$ forest transmitted flux fraction $F=e^{-\tau}$, where the density bias $b_{F}^{\delta}$ (response to large-scale overdensity) and the velocity bias $b_{F}^{\eta}$ (response to line-of-sight velocity gradient) are expressed as

$$
b_{F}^{\delta}=\left\langle\frac{d F}{d \delta}\right\rangle+\nu_{2}\left\langle\delta \frac{d F}{d \delta}\right\rangle, \quad b_{F}^{\eta}=\left\langle\tau \frac{d F}{d \tau}\right\rangle,
$$


with $\nu_{2}=34 / 21$. A third term is present in the biasing model of [20] that is proportional to the level of non-Gaussianities $f_{N L}$ in the primordial density fluctuations. In this study we assume that $f_{N L}=0$, and ignore this extra term.

The derivation in [20] ignores the shift in the overall scale caused by the long wavelength overdensity. For second order $\delta^{2}$ bias, this shift term comes as $d \ln \left[k^{3} P(k)\right] / 6 d \ln k$ [31], which should be compared to $\nu_{2}=34 / 21$. At the Ly $\alpha$ forest scale $k \sim 1 h / M p c k^{3} P(k)$ has a slope of approximately 0.6 , and if we smooth $\operatorname{Ly} \alpha$ forest with even smaller R (higher k) then it is even smaller. The correction is 0.1 relative to $34 / 21$, i.e. it is of order $6 \delta^{2}$ bias term. We will also continue to ignore this correction here.

\subsection{Biasing of a generic transformation}

In section 3.2 we have studied the effect of applying a generic analytic transformation to the Ly $\alpha$ forest transmitted flux fraction $g(F)=\sum_{m=1}^{\infty} a_{m} F^{m}$. Because the derivation of eq.4.1 in paper [20] can be applied to an arbitrary analytic function of $\delta$, we can thus compute the bias parameters of the tranformed field by replacing $F$ with $g(F)$ in eq.4.1:

$$
b_{g}^{\delta}=\left\langle\frac{d g(F)}{d \delta}\right\rangle+\nu_{2}\left\langle\delta \frac{d g(F)}{d \delta}\right\rangle, \quad b_{g}^{\eta}=\left\langle\tau \frac{d g(F)}{d \tau}\right\rangle .
$$

Furthermore, using the chain rule we can substitute the derivatives of $g(F)$ by the corresponding derivatives of $F$ multiplied by the derivative of the transformation itself (i.e., $\left.\frac{d g(F)}{d F}=\sum_{m=1}^{\infty} m a_{m} F^{m-1}\right)$. The biasing of $g(F)$ can then be expressed as

$$
b_{g}(\mu)=b_{g}^{\delta}+\left(f \mu^{2}\right) b_{g}^{\eta}=\left\langle\left(\sum_{m=1}^{\infty} m a_{m} F^{m-1}\right)\left(\frac{d F}{d \delta}+\nu_{2} \delta \frac{d F}{d \delta}\right)\right\rangle+f \mu^{2}\left\langle\left(\sum_{m=1}^{\infty} m a_{m} F^{m-1}\right) \tau \frac{d F}{d \tau}\right\rangle .
$$

As pointed out in [20], the velocity bias $b_{F}^{\eta}$ is completely determined by the probability distribution function (PDF) of the field, $p_{F}(F)$. This is also true for a generic transformation, where the velocity bias can be computed as:

$$
b_{g}^{\eta}=\sum_{m=1}^{\infty} m a_{m}\left\langle F^{m} \ln (F)\right\rangle=\sum_{m=1}^{\infty} m a_{m} \int_{0}^{1} d F p_{F}(F) F^{m} \ln (F) .
$$

The PDF can be computed both in the data and in hydrodynamic simulations, allowing for a test of the model presented in [20].

On the other hand, the computation of the density bias $b_{g}^{\delta}$ requires the derivative of the transmitted flux fraction with respect to the density field, $\frac{d F}{d \delta}$. In order to compute this value we have to assume an analytic relation $F(\delta)$, which will strongly affect the predicted value. In the next subsection, we will present the predictions for $b_{g}^{\eta}$ and $b_{g}^{\delta}$ using a particular model of $F(\delta)$.

\subsection{Bias computation with Fluctuating Gunn-Peterson Approximation}

The Fluctuating Gunn-Peterson Approximation (FGPA) suggests that the optical depth of Ly $\alpha$ has a simple dependence on the local density of the IGM:

$$
\tau=A(1+\delta)^{\alpha}
$$

Here, $\alpha=2-0.7(\gamma-1)=2-0.7 \frac{d(\ln \rho)}{d(\ln T)}$, where $\rho$ is the intergalactic gas density and $T$ is the temperature of the IGM, the amplitude $A$ is proportional to $T^{-0.7} \Gamma^{-1}$ where $\Gamma$ is the 
ionization rate by cosmic UV backgroud [32, 33]. In particular, $\alpha$ typically ranges from 1.6 to 2 [32]. The FGPA has been shown to be a good approximation at the relevant redshifts of interest for BAO measurements $2 \lesssim z \lesssim 3.5$. However, a caveat: eq.4.1 was derived in paper [20] on the basis of Taylor expansion

$$
\tau(\delta)=\sum_{n=0}^{\infty} \frac{\tau^{(n)}(0)}{n !} \delta^{n}
$$

where the convergence radius is 1 in FGPA, which implies the application of FGPA in our prediction is only valid for at most $|\delta| \leq 1$. For crude test, we do not restrict ourselves to this domain of convergence in the following study, but we are aware that this domain restriction of FGPA might be responsible for any discrepancy between the predictions and the measurements in section 4.3 .

Now, differiating $F$ with respect to $\delta$ according to eq.4.5 and defining the auxiliary function $r(F)=\alpha \ln (F)\left(\nu_{2}+\left(1-\nu_{2}\right)\left(-\frac{\ln (F)}{A}\right)^{-\frac{1}{\alpha}}\right)$, we can express the density bias of a generic transformation as

$$
b_{g}^{\delta}=\sum_{m=1}^{\infty} m a_{m}\left\langle F^{n} r(F)\right\rangle=\sum_{m=1}^{\infty} m a_{m} \int_{0}^{1} d F p_{F}(F) F^{m} r(F) .
$$

Finally, to compute eq.4.4 and eq.4.7, besides the PDF from data or from a simulation we can also use the theoretically approximated PDF in which the density field $\delta$ follows a log-normal distribution:

$$
1+\delta=e^{\left(\delta_{G}-\frac{\sigma^{2}}{2}\right)}, \quad p_{G}\left(\delta_{G}\right)=\frac{1}{\sqrt{2 \pi \sigma^{2}}} e^{-\frac{\delta_{G}^{2}}{2 \sigma^{2}}},
$$

where $p_{G}\left(\delta_{G}\right)$ is the PDF of the auxiliary Gaussianized field $\delta_{G}$.

\subsection{Testing the model with simulations}

In order to use the FPGA and the log-normal model for the density field, we need to choose a value for the parameters $\alpha, A$, and $\sigma$. Using the hydrodynamic simulations presented in section 2.3, we fit these parameters by comparing the model predictions and the simulation measurements of three different statistics: flux PDF $p_{F}(F)$, flux moments $\left\langle F^{n}\right\rangle$ and $\left\langle F^{n} \ln F\right\rangle$.

In this section we will focus on the results at $z=2$, but we study other redshift outputs in appendix B. The results are qualitatively similar, although in general we find the fits at lower redshift to be slightly better. In figure 6 we compare the flux PDF measured in the simulation with that predicted by the best fit model: $\alpha=1.22, A=0.31$ and $\sigma=1.72$. Notice that the best fit value of $\alpha$ is smaller than the typical range of $1.6<\alpha<2$.

Even though the first moments $\left\langle F^{n}\right\rangle$ are quite well fitted, as shown in figure 7, it is clear from figure 6 that no combination of parameters is able to reproduce the measured PDF, especially at the high flux end. Qualitatively, we find the following trends: (1) increasing $\alpha$ moves the moments $\left\langle F^{n}\right\rangle$ up and vice versa, but higher moments are more sensitive to the change of $\alpha$ than lower moments with the first moment $\langle F\rangle$ being almost immune to the change of $\alpha$; (2) increasing parameter $A$ readily moves all the moments $\left\langle F^{n}\right\rangle$ down by similar amount, and vice versa; (3) changing $\sigma$ has similar effect on the moments as changing $\alpha$, but the moments (especially the lower moments) are generally more sensitive to the change of $\sigma$ than in $\alpha$ case. 


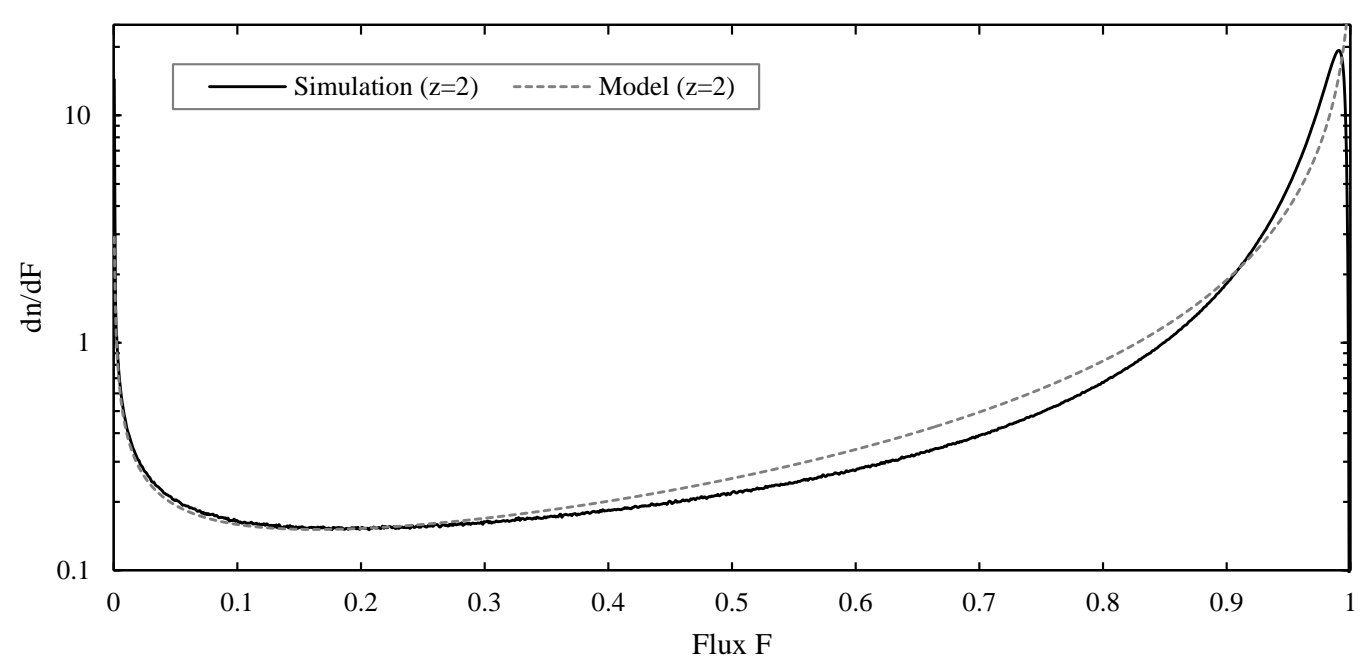

Figure 6. Comparison of the flux PDF measured in simulations with that predicted by the best fit parameters of the FGPA + log-normal model.

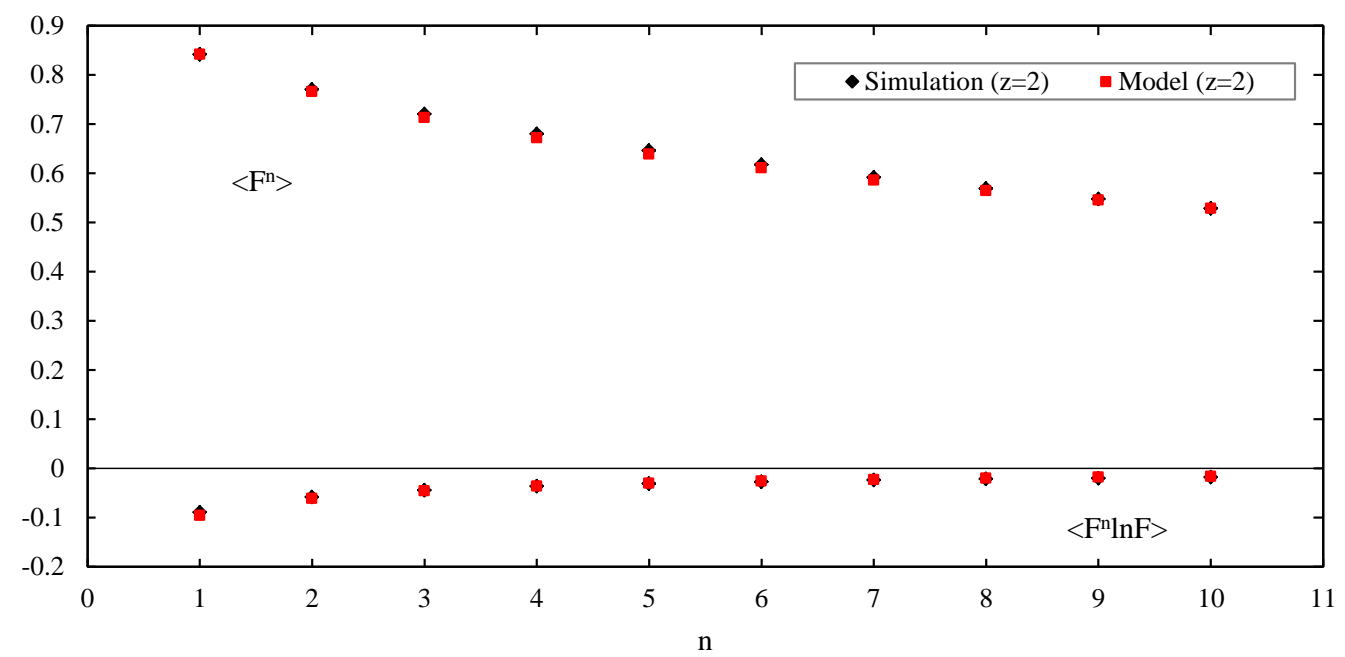

Figure 7. Comparison of the first flux moments in simulations with those predicted by the best fit parameters of the FGPA (upper panel), and of the averages of $\left\langle F^{n} \ln (F)\right\rangle$ relevant to the computation of velocity biases (lower panel).

As described above, in order to predict the value of the bias parameters we need to use a flux PDF. Since the best fit model does not reproduce very well the PDF measured in simulations, we will present two predictions for the bias parameter: one using the model $P D F$ and one using the simulation PDF.

In figure 8 we show the predictions for the bias parameters of $F, F^{2}, \ldots, F^{6}$, compared to the values measured in simulations at $z=2$, where the prediction using the model PDF is somewhat closer than the prediction using the measured PDF - however, as shown in appendix B, this is not the case in other redshifts. From the plots it is clear that while we are able to correctly predict the velocity bias for the first orders $\left(b_{F}^{\eta}, b_{F^{2}}^{\eta}, b_{F^{3}}^{\eta}\right)$, the model fails to reproduce the measured density bias even for the original field $b_{F}^{\delta}$. In fact, it is not surprising that the prediction for the density bias is worse than the prediction for the velocity bias, since the former not only depends on the PDF but also in the assumed relation $\tau(\delta)$. And as discussed in section 4.2 that the FGPA application is restricted by $|\delta| \leq 1$, this 


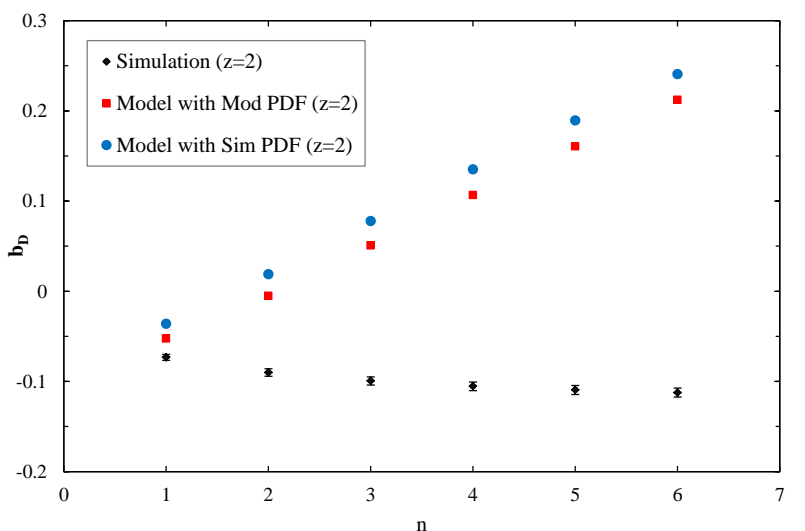

(a) Density bias $b_{F^{n}}^{\delta}$.

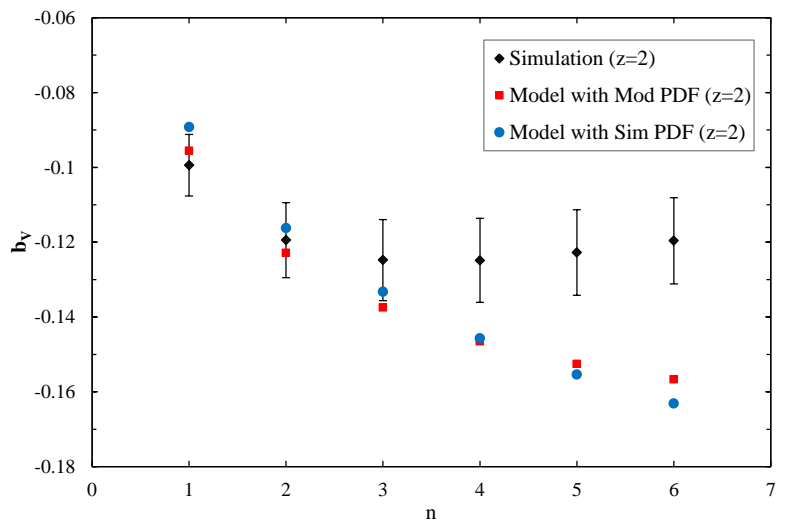

(b) Velocity bias $b_{F^{n}}^{\eta}$.

Figure 8. Comparison of predicted and measured biases at $z=2$. The blue and the red data points in both panels correspond to the parameter best-fitting values presented in the main text.

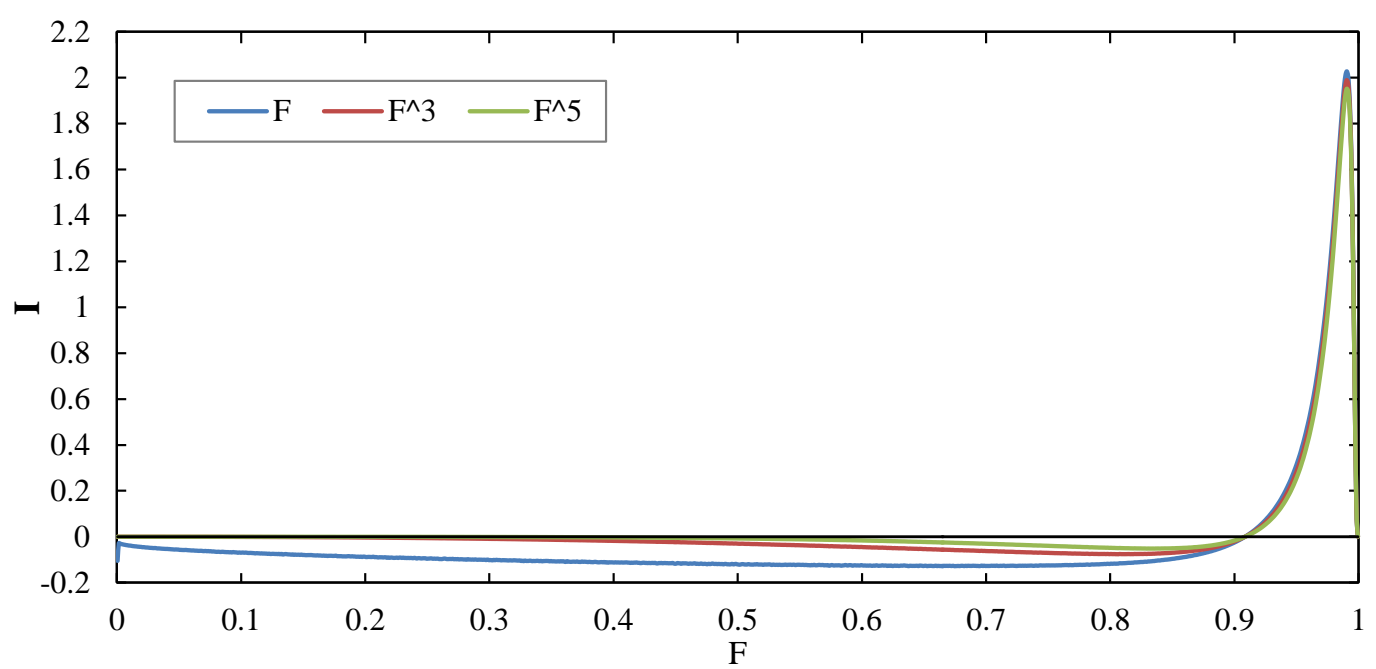

Figure 9. Plots of integrands $I=p_{F}(F) F^{n} r(F)$ in eq.(4.7) for density bias $b_{F}^{\delta}, b_{F^{3}}^{\delta}$ and $b_{F^{5}}^{\delta}$, where $p_{F}(F)$ is the simulation flux PDF.

domain restriction may be responsible for the poor predictions of density bias.

The prediction of the density bias for the higher order fields $F^{n}$ is particularly difficult, since it is completely dominated by the high $F$ end of the PDF, where the model performs poorly. This is clearly seen in figure 9 , where we show the integrand of equation 4.7 for three fields of interest: $F, F^{3}$ and $F^{5}$.

\section{Conclusions}

In the context of optimizing the measurement of the BAO scale in Ly $\alpha$ forest surveys, we have presented a study of the effect of non-linear transformations of the transmitted flux fraction $F \rightarrow g(F)$ on the expected signal-to-noise ratio of the measurement.

On the large scales relevant in a BAO measurement, the signal is proportional to the square of the large-scale biasing of the field. The noise, on the other hand, has several contributions. In the limit of being dominated by aliasing noise (equivalent to shot-noise in 
a galaxy survey), the noise is proportional to the amplitude of the one-dimensional power spectrum at low- $k$ limit. Under these assumptions, we study the signal-to-noise ratio for different transformations using hydrodynamic simulations.

The first transformation that we have studied is a monotonic relation $g(F)$ so that the final field has a Gaussian PDF. We have shown that the Gaussianized field obtained with this transformation would have $\mathrm{a} \approx 33 \%$ larger signal-to-noise ratio in the transverse BAO measurement, but $\mathrm{a} \approx 30 \%$ smaller ratio along the line of sight. The anisotropy of the gain can be explained by a significantly lower value of the redshift space distortion parameter $\beta$ in the Gaussianized field.

We have also studied the case of analytic transformations of the form $g(F)=\sum_{m=1}^{n} a_{m} F^{m}$, and presented results for generic quadratic $(n=2)$ and cubic $(n=3)$ transformations. In both cases, the angularly averaged maximum gain in signal-to-noise that we measure is rather small $(<5 \%)$.

Finally, we have extended the biasing model presented in [20] to describe the biasing of higher order field $F^{n}$. We have shown that while the model is able to describe the velocity bias $b^{\eta}$ reasonably well for the first orders $(n=1,2,3)$, the density bias $b^{\delta}$ is difficult to predict even for the original field $F$.

Our findings may be of use in attempts to optimize BAO signal to noise in realistic Ly $\alpha$ forest surveys. It is possible that one can use transformations such as Gaussianization to significantly improve the measurement of angular diameter distance $D_{A}$. One could perhaps envision that the measurement of $H$ along the line of sight is done using flux field itself, while measuring $D_{A}$ in the transverse direction would be done using Gaussianized field. For a more realistic estimate of the actual gains one should include measurement noise and resolution in the analysis, which was ignored here. These will depend on individual surveys and are beyond the scope of this paper, but should be explored further in the future.

\section{Acknowledgments}

This work is supported partly by the 2013 Summer Undergraduate Research Fellowship Program (SURF) at the University of California, Berkeley, and by NASA ATP grant NNX12AG71G. We would like to thank Nishikanta Khandai for providing the hydrodynamic simulation, and Anže Slosar, Patrick McDonald and Martin White for useful discussions.

\section{A Bias fitting procedures}

The first step towards measuring the bias parameters of the Ly $\alpha$ transmission flux fraction $F$ is to measure its 3D power spectrum. We measure band powers in a grid of 3 wavenumber bins limited by $0.0,0.4,0.8$ and $1.2 \mathrm{hMpc}^{-1}$, and in 4 bins in angular coordinate $\mu=k_{\|} / k$, limited by $0,0.25,0.5,0.75$ and 1 , for a total of $N=12$ bands.

We start by adding the norm of the relevant 3D Fourier modes (obtained with a FFT) within each of the $N$ bands, and treat these values as our data vector (or $\mathbf{P}_{\mathbf{o}}$ ). We then add the prediction from our fiducial model (described below) for each of the modes in the bands, and treat these as the theoretical prediction ( or $\mathbf{P}_{\mathbf{t}}$ ). We estimate the (diagonal) covariance $\mathbf{C}$ of the data vector by adding $2 \mathbf{P}_{\mathbf{t}}{ }^{2}$ for each mode within a band. We use these ingredients to compute a maximum likelihood estimator of the band power, where the likelihood $L$ is 
proportional to:

$$
L \propto \operatorname{det}(\mathbf{C})^{-1 / 2} \exp \left[-\frac{1}{2}\left(\mathbf{P}_{\mathbf{o}}-\mathbf{P}_{\mathbf{t}}\right)^{t} \mathbf{C}^{-1}\left(\mathbf{P}_{\mathbf{o}}-\mathbf{P}_{\mathbf{t}}\right)\right]
$$

We are interested in measuring the scale-independent density bias $b^{\delta}$ and the velocity bias $b^{\eta}$ parameters, which describe the clustering on linear scales. However, it is difficult to fit linear bias parameters in a box that is only $40 h^{-1} \mathrm{Mpc}$ wide, since there are very few Fourier modes that are in the linear regime. Therefore, we need to model the deviations from linear theory in the simulations, and marginalize over the free parameters these. Based in the description of large scale biasing of [34], we parameterize the deviations from linear theory with the following analytic model:

$$
P_{F}\left(k, k_{\|}\right)=\left[b^{\delta}\left(k, k_{\|}\right)+b^{\eta}\left(k, k_{\|}\right) f \frac{k_{\|}^{2}}{k^{2}}\right]^{2} P(k)+N\left(k, k_{\|}\right),
$$

with

$$
b^{\delta}\left(k, k_{\|}\right)=b_{\delta}^{0,0}+b_{\delta}^{2,0}(R k)^{2}+b_{\delta}^{0,2}\left(R k_{\|}\right)^{2}+b_{\delta}^{4,0}(R k)^{4}+b_{\delta}^{0,4}\left(R k_{\|}\right)^{4}+b_{\delta}^{2,2}(R k)^{2}\left(R k_{\|}\right)^{2},
$$

where $b^{m, n}$ are free parameters, and the equivalent for $b^{\eta}\left(k, k_{\|}\right)$and $N\left(k, k_{\|}\right)$. In the expressions above, $R=0.2 h^{-1} \mathrm{Mpc}$ is a typical non-linear scale, and $P(k)$ is the linear matter power spectrum in the simulation. In total, we have 18 free parameters, including 6 parameters describing the (shot) noise in the measurement, but we are only interested in the values of $b^{\delta}$ and $b^{\eta}$ after marginalizing over the other 16 parameters. We have made sure that our results do not vary significantly if we remove all terms with four order in $R$, or if we increase/reduce the non-linear scale by a factor of 2 .

In order to estimate the uncertainties in our measured 3D power, we need to have an initial guess of the clustering. In practice, we iterate between the measurement of the $3 \mathrm{D}$ power with the fitting of the bias parameters, until it converges (usually within $<5$ iterations).

\section{B Large-scale bias for $\mathrm{z}=2.4$ and 2.75}

In section 4 we presented the predictions for the large scale bias at redshift $z=2$. Here we present some results at redshifts $z=2.4$ and $z=2.75$, which are qualitatively similar to those at $z=2$.

The parameters in the FGPA are redshift dependent. At $z=2.4$, the best fit values are $\alpha=1.465, A=0.357$ and $\sigma=1.275$, while at $z=2.75$ we find $\alpha=1.383, A=0.511$ and $\sigma=1.175$. At different redshifts, simulation PDF (of flux) performs differently with respect to model PDF (of flux) for predicting the biases. As we have seen in figures 6 and figure 7 , at $z=2$ model PDF performs better than simulation PDF for both the density biases and the velocity biases. However, from figures 10 it is hard to distinguish which version of PDF works better in general at $z=2.4$, and from figures 11 it is obvious that simulation $\mathrm{PDF}$ outperforms model PDF at $z=2.75$. Overall, observation suggests at high redshift simulation PDF performs better than model PDF, and vice versa. 


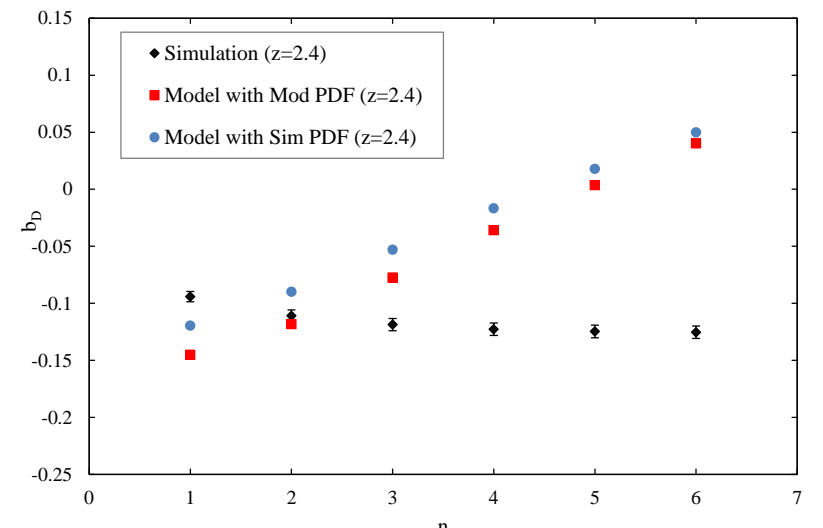

(a) Basis density biases $b_{F^{n}}^{\delta}$.

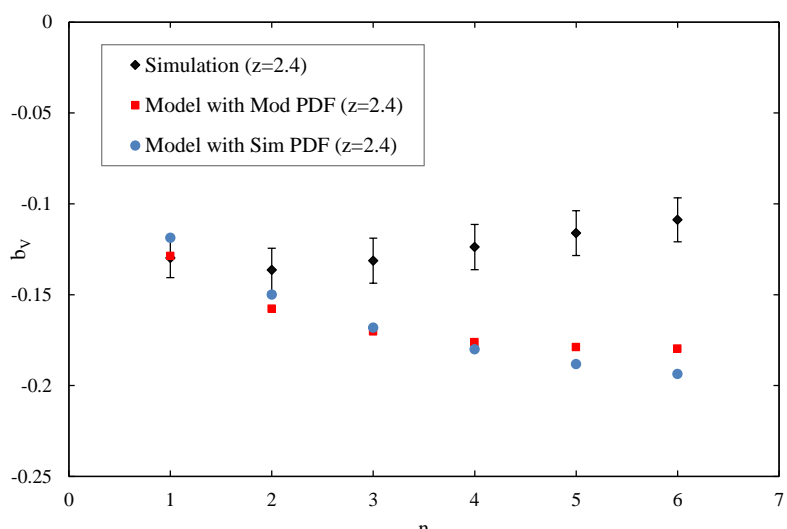

(b) Basis velocity biases $b_{F^{n}}^{\eta}$.

Figure 10. Comparison of predicted and measured basis biases at $\mathrm{z}=2.4$.

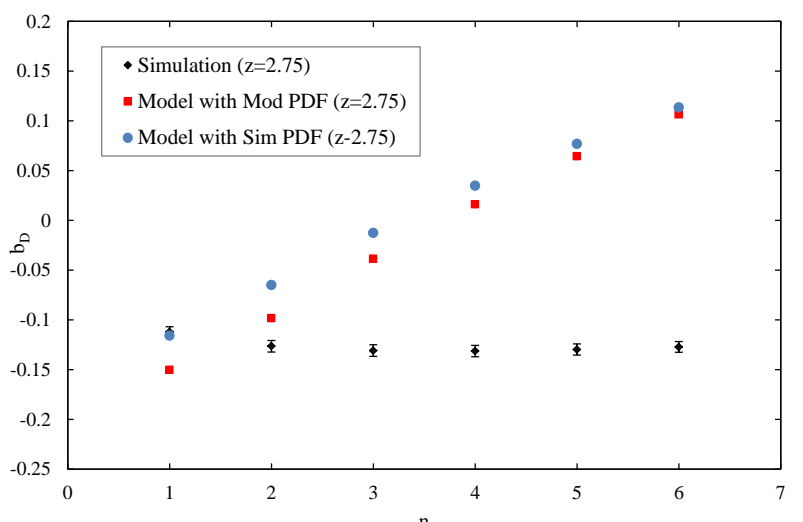

(a) Basis density biases $b_{F^{n}}^{\delta}$.

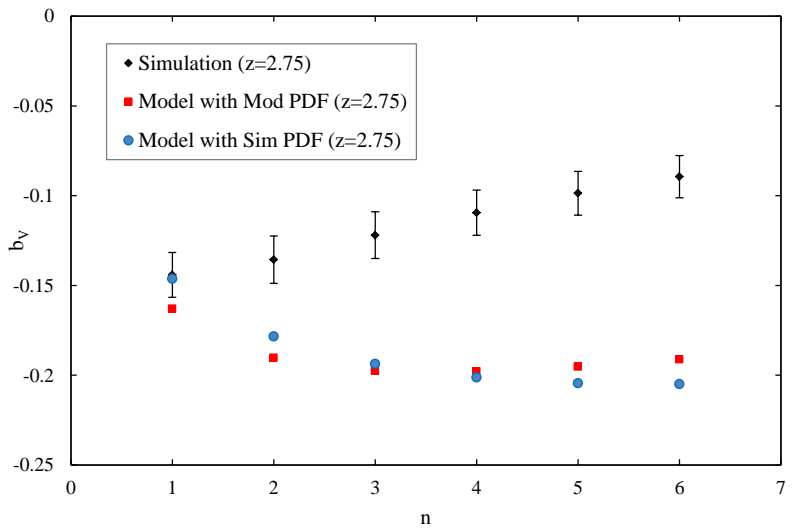

(b) Basis velocity biases $b_{F^{n}}^{\eta}$.

Figure 11. Comparison of predicted and measured basis biases at $\mathrm{z}=2.75$.

\section{References}

[1] D.H. Weinbeg, et al. Observational probes of cosmic acceleration, Physics Report 530 (2013) 87.

[2] H.J. Seo and D.J. Eisenstein. Probing Dark Energy with Baryonic Acoustic Oscillations from Future Large Galaxy Redshift Surveys, The Astrophysical Journal 598 (2003) 720.

[3] L. Anderson, et al. The clustering of galaxies in the SDSS-III Baryon Oscillation Spectroscopic Survey: baryon acoustic oscillations in the Data Releases 10 and 11 Galaxy samples, Monthly Notices of the Royal Astronomical Society.441 (2014) 24.

[4] S. Cole, et al. The 2dF Galaxy Redshift Survey: power-spectrum analysis of the final data set and cosmological implications, Monthly Notices of the Rolyal Astronomical Society 362 (2005) 505.

[5] C. Blake, et al. The WiggleZ Dark Energy Survey: mapping the distance-redshift relation with baryon acoustic oscillations, Monthly Notices of the Royal Astronomical Society 418 (2011) 1707.

[6] D.J. Eisenstein, et al. Detection of the Baryon Acoustic Peak in the Large-Scale Correlation Function of SDSS Luminous Red Galaxies, The Astrophysical Journal 633 (2005) 560. 
[7] N.G. Busca, et al. Baryon acoustic oscillations in the Lyd forest of BOSS quasars, Astronomy ES Astrophysics 552 (2013) A96.

[8] A. Font-Ribera, et al. Quasar-Lyaman a forest cross-correlation from BOSS DR11: Baryon Acoustic Oscillations, JCAP 05 (2014) 027.

[9] A. Slosar, et al. Measurement of Baryon Acoustic Oscillations in the Lyman-alpha Forest Fluctuations in BOSS Data Release 9, JCAP 04 (2013) 026.

[10] T. Delubac, et al. Baryon Acoustic Oscillations in the Lya forest of BOSS DR11 quasars, Astronomy \&3 Astrophysics . arXiv:1404.1801. 2014.

[11] M. Rauch. The Lyaman Alpha Forest in the Spectra of Quasistellar Objects, Annu.Rev.Astron.Astrophys 35 (1998) 267.

[12] A.A. Meiksin. The physics of the intergalactic medium, Reviews of Modern Physics 81 (2009) 1405.

[13] R.A.C. Croft, et al. Recovery of the Power Spectrum of Mass Fluctuations from Observations of the Lya Forest, The Astrophysical Journal 495 (1998) 44.

[14] R.A.C Croft, et al. The Power Spectrum of Mass Fluctuations Measured from the Lyo Forest at Redshift $z=2.5$, The Astrophyiscal Journal 520 (1999) 1.

[15] P. McDonald, et al. The Observed Probability Distribution Function, Power Spectrum, and Correlation Function of the Transmitted Flux in the Lya Forest, The Astrophysical Journal 543 (2000) 1.

[16] M.C. Neyrinck, et al. Rejuvenating the Matter Power Spectrum: Restoring Information with a Logarithmic Density Mapping, The Astrophysical Journal Letters 698 (2009) L90.

[17] H.J. Seo, et al. Re-capturing Cosmic Information, The Astrophysical Journal Letters $\mathbf{7 2 9}$ (2011) L11.

[18] B. Joachimi, et al. Cosmological information in Gaussianized weak lensing signals, Monthly Notices of the Royal Astronomical Society 418 (2011) 145.

[19] J.Carron and I. Szapudi. Sufficient observables for large-scale structure in galaxy surveys, Monthly Notices of the Royal Astronomical Society 439 (2014) L11.

[20] U. Seljak. Bias, redshift space distortions and primordial nongaussianity of nonlinear transformations: application to Ly- $\alpha$ forest, Journal of Cosmology and Astroparticle Physics 03 (2012) 004.

[21] J.E. Bautista, et al. Mock Quasar-Lyman- $\alpha$ Forest Data-sets for the SDSS-III Baryon Oscillation Spectroscopic Survey, JCAP (2015). arXiv: 1412.0658.

[22] A. Font-Ribera, et al. Generating mock data sets for large-scale Lyaman- $\alpha$ forest correlation measurements, JCAP 01 (2012) 001.

[23] Z. Lukić, et al. The Lyman $\alpha$ forest in optically thin hydrodynamical simulations, Monthly Notices of the Royal Astronomical Society 446 (2014) 3697.

[24] N. Kaiser. Clustering in real space and in redshift space, Monthly Notices of the Royal Astronomical Society 227 (1987) 1.

[25] P. McDonald and D.J. Eisenstein. Dark energy and curvature from a future baryonic acoustic

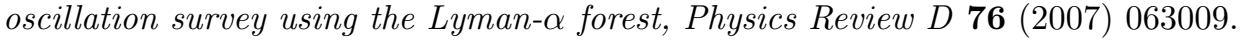

[26] M. McQuinn and M. White. On Estimating Lya Forest Correlations between Multiple Sightlines, Mon.Not.Roy.Astron.Soc. 415(2011) 2257.

[27] C. Stark, et al. The Lyman- $\alpha$ Forest in SPH and Eulerian Hydrodynamic Simulations. In preparation. 
[28] C.A Faucher-Giguére, et al. A New Calculation of the Ionizing Background Spectrum and the Effects of He II Reionization, the Astrophysical Journal 703 (2009) 1416.

[29] P. McDonald, et al. The Lyman- $\alpha$ Forest Power Spectrum from the Sloan Digital Sky Survey, The Astrophysical Journal 163 (2006) 80.

[30] D.H. Weinberg. Reconstructing primordial density fluctuations. I - Method, Monthly Notices of the Royal Astronomical Society 254 (1992) 315.

[31] B.D. Sherwin and M.Zaldarriaga. Shift of the baryon acoustic oscillation scale: A simple physical picture, Physical Review D 85 (2012) 103523.

[32] D.H. Weinberg, et al. The Lyman- $\alpha$ Forest as a Cosmological Tool, AIP Conf. Proc. 666 (2013) 157.

[33] R. Davé. Simulations of the Intergalactic Medium. arXiv: astro-ph/0311518v2. 2003.

[34] P. McDonald and A. Roy. Clustering of dark matter tracers: generalizing bias for the coming era of precision LSS, JCAP 08 (2009) 020. 